\title{
Civilisations
}

Revue internationale d'anthropologie et de sciences

humaines

42-1 | 1993

Amérique latine-Europe

\section{Les relations de l'Amérique latine et des Caraibes avec l'Europe communautaire : carrefour et défis}

\section{Veronica Loynaz}

\section{OpenEdition \\ Journals}

Édition électronique

URL : http://journals.openedition.org/civilisations/2113

DOI : 10.4000/civilisations. 2113

ISSN : 2032-0442

\section{Éditeur}

Institut de sociologie de l'Université Libre de Bruxelles

\section{Édition imprimée}

Date de publication : 1 novembre 1993

Pagination : 221-235

ISBN : 0009-8140

ISSN : 0009-8140

\section{Référence électronique}

Veronica Loynaz, «Les relations de l'Amérique latine et des Caraïbes avec l'Europe communautaire carrefour et défis », Civilisations [En ligne], 42-1 | 1993, mis en ligne le 30 novembre 1996, consulté le 19 avril 2019. URL : http://journals.openedition.org/civilisations/2113; DOI : 10.4000/ civilisations. 2113

Ce document a été généré automatiquement le 19 avril 2019.

(c) Tous droits réservés 


\title{
Les relations de l'Amérique latine et des Caraibes avec l'Europe communautaire: carrefour et défis
}

\author{
Veronica Loynaz
}

1 Les liens entre Latino-Américains et Européens ont été marqués par des mouvements oscillatoires, stimulés ou freinés, suivant les vents qui soufflent du colosse nordaméricain. Pour entamer toute réflexion sur les relations entre l'Amérique latine et l'ancien continent, il faut donc tenir compte des variables qui forment ce que l'on appelle le «triangle atlantique », puisque l'histoire ancienne et les faits récents indiquent que la variable nord-américaine pèse d'un poids écrasant sur les desseins que tentent de réaliser les divers acteurs européens sur la scène latino-américaine. Prétendre faire abstraction de cette vérité équivaudrait à adopter une position non réaliste, non conforme aux nouveaux signes de l'actualité. L'histoire la plus récente nous montre que, dans leurs tentatives pour se lier aux latino-américains, les Euro-occidentaux se sont heurtés aux mêmes fissures, aux mêmes érosions que celles qu'a connu l'hégémonisme nordaméricain, il y a des années, dans les pays situés au sud du Rio Bravo.

2 Les 10 ou 20 dernières années ont été les témoins de changements inimaginables, à une vitesse vertigineuse qui anéantit la sérénité et la réflexion nécessaires à une meilleure analyse. Il faut donc définir les relations euro-latino-américaines dans ce nouveau contexte international, à la lumière du nouvel ordre mondial qui se présente sous les traits bien définis d'un accroissement de l'inégalité entre le Nord et le Sud.

3 Les deux acteurs impliqués dans ce sous-système de relations sont soumis aux avatars de la nouvelle réalité à affronter. Pour le meilleur ou pour le pire, tant l'Europe communautaire que l'Amérique latine et les Caraïbes sont secouées par les nouveaux signes des temps. Le contexte international apparaît aujourd'hui marqué par une forte tendance à la globalisation de l'économie et à la formation de grands groupes régionaux, d'espaces géoéconomiques menés par les États-Unis, le Japon et l'Europe communautaire. Selon cette nouvelle tendance à la formation de megamarchés, chacun d'eux tente de 
s'allier aux régions voisines pour former des espaces économiques, des sphères d'influence et de réalisation qui les confortent dans leur concurrence, et. ainsi, favoriser un recrutement sélectif du monde sous-développé dans la tripolarité économique des alliances occidentales.

Dans ce nouveau cadre mondial, les puissants d'hier et d'aujourd'hui décident des grandes lignes d'action. Outre le pouvoir économique, politique, technologique et militaire dont ils jouissent dans leurs domaines continentaux respectifs, ils disposent de la force et des mécanismes suffisants pour imposer leurs critères et leurs intérêts dans les divers organismes internationaux tels que le GATT, le FMI, la Banque mondiale et même l'ONU. D'autre part, et en contrepartie, le monde sous-développé en général, et l'Amérique latine en particulier, est encore plongé dans une profonde crise qui a provoqué un grave retard économique et social, la désarticulation de la production, la marginalisation sur le marché international, l'intégration transnationale et la désintégration nationale, la décapitalisation financière et le retard technologique, scientifique et culturel. Ce monde relégué réclame la reconnaissance de ses problèmes et de ses difficultés, un meilleur traitement et une plus grande participation dans les nouvelles relations économiques et politiques internationales. Mais ces revendications sont dédaignées et ignorées, et le conditionnement politique s'accentue de jour en jour en vue d'un traitement préférentiel ou, en dernière instance, de la sélection pour le « recrutement $»$ actuel $^{1}$.

5 Face à la situation internationale contemporaine, il convient de se demander - et c'est là une préoccupation de première importance au moment d'entamer l'analyse des relations entre latino-américains et européens - quelle est la priorité accordée au sous-continent dans la pyramide des intérêts économiques et politiques de l'Europe communautaire concernant ses relations de pôle avec les États-Unis et le Japon, le reste des pays européens à l'Est et à l'Ouest, l'ex-Union Soviétique, les pays de la Méditerranée, les économies plus développées et dynamiques d'Asie, la Chine, et ses engagements institutionnels liés à la convention de Lomé pour les pays $\mathrm{ACP}^{2}$. Malheureusement, si nous nous fions aux chiffres, les conclusions sont peu encourageantes. En marge de tous les éléments endogènes et exogènes qui séparent les deux régions, il existe indiscutablement une volonté politique soutenue par des intérêts économiques lancés à la recherche de nouvelles voies pour tenter d'éviter le croisement actuel de ces relations et ainsi, innover en la matière.

\section{Les voies du carrefour}

\section{Les défis européens : « l'europhorie ». Maastricht 1993}

6 L'Europe communautaire se trouve aujourd'hui immergée dans son grand projet d'unité européenne ; autrement dit, elle cherche à réaliser ses potentialités par l'union si souvent planifiée et ayant provoqué la mélancolie des " pères de la construction européenne », les promoteurs des idées de fédéralisme sur le vieux continent.

7 L'Europe des années 90 est le théâtre d'une contradiction très singulière. Si, d'un côté, l'Ouest européen tente d'élaborer une construction intégratrice et unificatrice pour une Europe sans frontière, par la création de l'espace économique européen et ses diverses dimensions politiques et sociales, d'autre part, en Europe de l'Est et dans l'ex-URSS, s'agitent les passions nationalistes, prévalent les forces centrifuges, le fantasme de la 
division nationaliste; les déséquilibres économiques et politico-sociaux secouent cette sous région. L'intégration, matérialisée par la Communauté économique européenne (CEE), fut un observateur enthousiaste de l'échec et de la chute du système socialiste européen, alors qu'aujourd'hui elle tente d'inclure dans sa sphère d'influence un Est européen balkanisé par les luttes intestines et les nationalismes exacerbés.

Toutefois, ces dernières années, l'Europe a connu une renaissance de ce que l'on a appelé «l'europhorie ». La CEE a lancé un ambitieux projet d'unité économique et politique qui, après les accords du dernier sommet de Maastricht, a pris une forme plus précise, adopté des objectifs plus affinés et mieux définis. Autour de la CEE se forment progressivement les divers cercles concentriques de relation avec le reste des États européens de l'Ouest et de l'Est. Sans aucun doute, malgré ses détracteurs, ses progrès lents et ses marches arrières, l'Europe devient un pôle au niveau international, et la devise «l'union fait la force » influence toutes les sociétés européennes dans toutes leurs dimensions.

9 Ainsi, on est parvenus à approfondir l'intégration économique en élargissant le projet d'espace économique européen aux pays membres de l'Association européenne de libreéchange (AELE) , marché auquel adhérera progressivement l'ensemble de l'Europe occidentale. Sans doute l'Europe est-elle prise dans la dialectique de la réussite de son unité et de son élargissement. Tout ce processus se base sur l'homogénéisation des marchés pour atteindre les objectifs déjà signalés. En réalité, le projet communautaire de l'Europe représente une expérience singulière dans laquelle des souverainetés sont déléguées à des institutions supranationales.

10 L'Europe communautaire doit relever plusieurs défis sur le plan intérieur, la marche vers le marché de 93 est solidement posée et se traduit par une stratégie cohérente et irréversible, l'eurosclérose a été remplacée par l'euro-optimisme. En dépit du «non » danois, Maastricht poursuit sa route polémique. Sur le plan extérieur, rien de ce qui se passe dans l'Est européen ne peut laisser les États communautaires indifférents.

11 La résultante de cet intéressant processus conduit à une conclusion fondamentale : les Européens plongés dans leur construction régionale, se concentrent de plus en plus sur leur débat interne et leurs grands objectifs et laissent de côté les difficultés non liées à leurs buts eurocentriques. L'histoire de la Communauté confirme que, lorsqu'elle tente d'approfondir son processus d'intégration, d'une certaine manière elle s'enferme en ellemême; ce fut son attitude dans les années 60 , au moment où elle a commencé à former son union douanière. Actuellement, il y a une certaine analogie avec cette étape. Ces préoccupations lèsent indiscutablement ses relations avec d'autres parties du monde, et l'Amérique latine n'est pas parmi les régions prioritaires. Cette situation représente certainement un facteur négatif pour les relations avec l'Amérique latine.

12 Un autre élément endogène qui influe négativement sur les relations euro-latinoaméricaines, et est étroitement lié à la tendance à la formation de blocs régionaux et à l'essor du projet d'unité européenne, concerne les trois grands pôles économiques qui cherchent à se distribuer les zones naturelles et voisines d'influence et de réalisation de leurs projets respectifs. Selon cette logique, tout semble indiquer que l'Europe communautaire assumera le rôle principal dans l'Est européen, et, malheureusement pour l'Amérique latine et les Caraïbes, elles subissent le poids des États-Unis avec un accent marqué de l'axe vertical interaméricain qui s'est matérialisé, aux temps du nouvel ordre mondial, par l'Initiative pour les Amériques lancée par le président Bush. Dans cette logique d'action, cet élément agirait comme facteur défavorable au développement des relations, au-delà des perspectives d'élargissement. 
13 Mais il y a l'autre côté de la médaille, c'est-à-dire les facteurs de cette architecture européenne que l'on veut dessiner. Ils pourraient devenir des facteurs positifs, ou du moins contribuer au rapprochement des deux régions. Dans le processus de redéfinition interne, d'élargissement des possibilités, de recherche d'un espace autonome de prise de décisions propre aux diverses institutions communautaires pour avoir plus de poids dans l'échafaudage institutionnel de la Communauté, on estime qu'il y a actuellement une sensibilité institutionnelle relativement plus importante vis-à-vis de l'Amérique latine, qui se traduit manifestement par des projets spécifiques, de nouveaux liens et des réglementations communautaires concrètes.

Pour n'en citer que quelques-uns, mentionnons le dialogue économique et politique de la CEE avec le Groupe de Rio, les traditionnelles rencontres de San José, avec des actions non spectaculaires mais novatrices dans la coopération communautaire, le traitement préférentiel et conjoncturel, dans le commerce andin et centre-américain en tant que formule d'aide à la lutte contre la drogue, pour certains produits originaires de cet ensemble de pays (il s'étendra exceptionnellement pendant 4 ans), les diverses résolutions du Parlement européen dans des matières qui concernent spécifiquement l'Amérique latine et les Caraïbes ; enfin, toute l'Amérique latine est couverte par une série d'accords bilatéraux et multilatéraux divers : le bilatéralisme sélectif caractéristique des relations entre les deux régions y est prépondérant. L'Amérique latine est la région qui a passé, avec la Communauté, la plus grande quantité d'accords de la troisième génération, c'est-à-dire les accords les plus avancés avant le statut d'association ${ }^{3}$. Dans la hiérarchie interne de la Communauté, et étant donné qu'il est risqué de vouloir schématiser les diverses positions communautaires des pays membres les plus importants, moyennant une incidence négative ou positive, on pourrait présenter l'ordre de priorités suivant :

1. ces derniers temps, la Grande-Bretagne et la France sont apparues dans le cadre communautaire comme les pays les plus réticents aux résolutions et aux actions en faveur de l'Amérique latine ;

16 2. l'Allemagne, la Hollande et la Belgique constituent un autre pôle dont la réticence ou non peut varier en fonction du thème analysé ;

17 3. l'Espagne et l'Italie sont les promoteurs d'initiatives favorables à l'élargissement et à la diversification des relations avec l'Amérique latine.

\section{Le dilemme latino-américain}

Le sous-continent présente un dilemme récurrent, mais essentiel au moment d'analyser tout lien avec cette région: la situation économico-sociale critique et appauvrie dans laquelle il se trouve plongé. L'Amérique latine vit toujours dans une stagnation relative et a connu un nouveau recul du revenu par habitant ; la dette frappe toujours l'ensemble des pays; l'inflation est irrépressible; l'augmentation du chômage et du sous-emploi est insoutenable. La croissance globale latino-américaine n'a été que de 1,4\% en 1989 et de - 0,5 \% en 1990 ; le revenu par habitant a diminué pour la troisième année consécutive : il est revenu à son niveau de 1977, et la décennie perdue pour le monde sous-développé, et particulièrement pour l'Amérique latine, a coïncidé avec la plus longue période d'expansion de l'économie internationale ${ }^{4}$.

Dans le contexte régional, de nombreuses voix se sont élevées - dont celle du SELA - pour souligner la vulnérabilité actuelle de l'Amérique latine face aux changements 
économiques mondiaux. Le modèle d'économie sociale de marché et les politiques d'ajustement, basées sur les recettes néo-libérales, ont des conséquences désastreuses pour les sociétés latino-américaines. Il est indispensable que l'Amérique latine passe d'une insertion passive dans l'économie internationale à une participation active, différente.

Dans ce contexte difficile, et d'un point de vue géopolitique, l'intensification des relations avec l'Europe est importante pour la région latino-américaine. Il ne s'agit pas seulement d'élargir les possibilités d'un équilibre politique avec le monde et de tempérer la force que pourrait avoir une dépendance excessive par rapport au marché nord-américain. La recherche d'un éventail diversifié d'alternatives de coopération est indispensable pour l'Amérique latine et les Caraïbes si elles veulent atteindre un niveau suffisant d'autonomie dans la prise des décisions relatives à leur développement. Les conditions actuelles ne semblent pas les plus adéquates pour que la CEE entreprenne une large renégociation de ses relations avec l'Amérique latine et les Caraïbes. La partie latinoaméricaine manque encore, malgré les éléments adverses, de la capacité de gestion et d'action. Cela correspond, dans le contexte latino-américain, à l'idée que l'unité et l'intégration latino-américaines sont nécessaires en tant que formule pour accroître la capacité de négociation du sous-continent.

21 Il convient de souligner que les projets latino-américains d'intégration sont passés par diverses étapes et que leurs particularités varient selon les sous régions et les pays impliqués. Mais ils ont en commun d'avoir perdu leur dynamisme et que leur réalisations ont une portée très limitée ; malheureusement, l'idée de l'unité et de l'intégration latinoaméricaines a été utilisée comme figure rhétorique dans le discours politique de la région. Une relance de ces projets s'impose aujourd'hui pour tenter d'arriver à des résultats concrets visant une meilleure insertion internationale.

L'Amérique latine est hétérogène. On y trouve des inégalités économiques marquantes, des projets et des relations particulières avec certaines puissances du Nord, au détriment des liens inter-latino-américains. Toutefois, un débat s'ouvre, un espace plus concret se dessine, étant donné la nécessité inéluctable d'atteindre l'unité avant le carrefour et d'essayer de répondre en tant que région - ou au moins en termes sous-régionaux - aux problèmes et aux obstacles que présentent la globalisation et la régionalisation des relations économiques et politiques interaméricaines. C'est un moment particulièrement important pour l'Amérique latine ; dans ce panorama, et avec de tels présupposés, il faut envisager toute possibilité de nouvelles relations entre Européens et Latino-américains.

D'autre part, dans l'optique des profils nationaux, on observe qu'un intérêt renouvelé a été suscité dans certains pays latino-américains en faveur du renforcement des relations avec l'Europe. De manière synthétique, on pourrait proposer le bilan suivant :

Mexique. On remarque une action diplomatique intense. C'est l'un des pays latinoaméricains qui a suscité le plus d'intérêt et d'expectatives dans les milieux européens. Diverses modalités de relations et de contacts ont été mises en pratique.

Chili. Le changement politique a amené une totale réinsertion diplomatique du pays en Europe. La situation économique relativement bonne du Chili, dans le contexte latinoaméricain, le place en position favorable pour resserrer ses liens avec l'Europe, grâce à une politique pragmatique et réaliste qui met l'accent sur le commerce et les investissements. 
Brésil. Le principal partenaire latino-américain de l'Europe ces deux dernières années a maintenu un profil assez bas dans ses relations avec l'Europe, entre autres, à cause des gros problèmes économiques internes qu'il connaît.

Argentine. On observe ces dernières années un certain changement de ton dans les importantes relations qu'elle entretient avec l'Europe, et tout vise à un nouveau pragmatisme, plus discret sur les appels à la solidarité et les récriminations mutuelles.

Venezuela. On remarque un intérêt européen, déterminé entre autres par ses considérables ressources énergétiques. De même, on constate que l'actuel président, Carlos Andrés Pérez, malgré la crise dans laquelle est plongé le pays, s'est montré très actif pour développer ses traditionnelles bonnes relations avec des leaders politiques de l'ancien continent.

Bolivie et Colombie. Elles ont fait l'objet d'intérêt et d'actions exceptionnelles et conjoncturelles, avec traitement préférentiel, dans la perspective de la lutte contre le trafic de drogue ${ }^{5}$.

\section{Le paradoxe des relations euro-latino-américaines}

Les relations euro-latino-américaines en sont encore à un stade d'indétermination et de totale insatisfaction. Évidemment, le sous-système de relations euro-latino-américaines connaît un déséquilibre entre des relations positives et vastes sur le plan politique et, d'autre part, une stagnation et une détérioration des rapports économiques, dans une perspective très sombre.

Dans sa relation avec l'Europe communautaire, l'Amérique latine a développé une insertion qui ne la favorise pas pour l'avenir. L'Amérique latine exporte principalement des produits agricoles, des matières premières et semi-manufacturées, ce qui ne permet pas le développement. De plus, si l'on considère la détérioration galopante des termes de l'échange, la situation inégale et critique qui en découle devient manifeste. L'Amérique latine n'est pas une région prioritaire pour les objectifs et la stratégie de l'Europe communautaire vis-à-vis du Tiers-Monde.

Malgré les reproches et les incompréhensions mutuelles, le débat actuel entre Européens et Latino-américains est parvenu à dépasser pour une bonne part la perception de ces relations dans l'optique de profils différenciés sur la manière dont nous nous voyons mutuellement. Avec davantage de réalisme, on a aujourd'hui abandonné l'idée qui pesait lourdement sur les rencontres pour trouver un interlocuteur valable et unique de région à région. Il existe un sens plus pragmatique. On renonce aux positions grandiloquentes qui ne mènent à aucun rapprochement réel et effectif. Les nouvelles relations que l'on prétend établir visent à potentialiser les contacts sous-régionaux ou certains groupes d'intérêts partiels ou spécifiques. C'est le cas du Groupe de Rio, expression d'une concertation politique naissante au niveau latino-américain; ou de l'ensemble des pays centre-américains qui ont fait l'objet d'une attention particulière en raison d'un conflit d'importance internationale durant plusieurs années; ou encore du Groupe Andin ou Mercosur, qui dénote une orientation nouvelle ou une relance de l'intégration latinoaméricaine. On essaie de renforcer les liens avec les processus d'intégration latinoaméricaine, en dépit de toutes leurs difficultés.

Ce panorama est tissé sans abandonner le traditionnel bilatéralisme sélectif, guidé et orienté par des intérêts économiques particuliers dans les pays latino-américains de plus grand développement relatif, ou par des motifs politiques en soutenant quelques-unes des 
valeurs essentielles pour le monde occidental, comme, par exemple, les transitions démocratiques de certains pays latino-américains. Ces motivations de type politique ont évidemment un sens et une durée très conjoncturels, et elles disparaissent lorsque ce moment particulier a pris fin.

Dans les relations Europe-Amérique latine, le rapprochement politique progressif - qui a été intense - s'est traduit, entre autres, par un puissant resserrement de liens diplomatiques et de coopération politique à divers niveaux et dans de nombreuses activités de caractère varié. On observe un vaste réseau d'interrelations sous-étatiques établies, entre autres, entre organisations non gouvernementales, le développement manifeste des internationales de partis, composées majoritairement aujourd'hui de partis européens et latino-américains. De même, on remarque le dialogue politique de haut niveau avec des organismes latino-américains qui, dans une certaine mesure, sont représentatifs de la concertation politique se déroulent dans le sous-continent ${ }^{6}$.

Il est important de souligner, sur le plan politique, que, depuis 1991, l'Espagne, pays communautaire, s'est lancée dans une intense activité qui culmine lors de la tenue du premier sommet Ibéroaméricain à Guadalajara, le deuxième ayant lieu à Madrid (1992). Sans vouloir surestimer les résultats concrets de ces réunions, disons qu'elles constituent un forum important de concertation politique, dans la perspective ibérique aussi bien que pour le côté latino-américain, puisqu'elles représentent pour les plus hauts dirigeants latino-américains une occasion unique de se réunir en dehors de toute présence nordaméricaine.

Pour l'Espagne, il s'agit essentiellement de canaliser des liens plus étroits avec les pays latino-américains et de tenter de tenir son rôle de "pont » entre l'Amérique latine et les autres pays communautaires. L'idée que l'Espagne agisse comme tel a suscité une certaine méfiance dans plusieurs secteurs latino-américains et n'a pas été soutenue avec tout l'enthousiasme espéré par le gouvernement espagnol. En fonction de cette réserve latinoaméricaine, l'attitude de l'Espagne a été qualifiée de paternaliste et de rhétorique, et l'Amérique latine a souligné qu'elle n'avait pas besoin de la tutelle espagnole pour faire valoir ses intérêts vis-à-vis du reste de l'Europe communautaire.

31 Le panorama change si l'on analyse le point de vue économique. Dans les relations économiques, on observe une détérioration soutenue et progressive du commerce; il y a aujourd'hui entre les deux parties une "marginalité réciproque», comme le souligne l'analyste Manfred Whilhelmy ${ }^{7}$.Le problème devient flagrant lorsque l'on tient compte de la situation critique du sous-continent et de sa nécessité de s'intégrer de manière active à l'ordre économique actuel. Ces frustrations au plan commercial représentent l'un des multiples aspects de la stagnation des relations économiques birégionales.

Certains éléments ont une forte incidence négative sur les relations euro-latinoaméricaines, parmi lesquels nous pouvons citer le protectionnisme, la politique agricole commune de la Communauté, la position de la CEE vis-à-vis de la dette extérieure latinoaméricaine et le manque de priorité de l'Amérique latine dans la coopération de la CEE. D'autre part, les implications du marché unique européen - et au-delà de l'espace économique européen à l'horizon de 1993 - ne peuvent être analysées de manière statique, mais doivent l'être dans la dynamique propre de ce processus lorsqu'il commencera à fonctionner dans toute sa dimension. Il y aura des affectations diverses pour certains produits latino-américains. Le débat entre l'Europe forteresse contre l'Europe des chances ouvertes est encore latent. Un élément s'intégrant au nouveau contexte international est le facteur compétitif, qui a introduit dans le sous-système de 
relations entre l'Europe et l'Amérique latine la présence de l'Europe de l'Est, ce qui complique la situation et met à l'ordre du jour le débat entre l'Est et le Sud.

Tout semble indiquer que, au cours de cette nouvelle étape, marquée par des « relations internationales de marché » qui remplacent ce que l'on a appelé la diplomatie des partis, l'Amérique latine doit revoir radicalement la stratégie à suivre dans ses relations avec l'Europe. Dans cette nouvelle étape, les principaux acteurs seront les agents économiques, ainsi que les responsables gouvernementaux des politiques économiques. Il devient indispensable d'établir entre les pays latino-américains un niveau de concertation bien supérieur à celui qui existait jusqu'à présent ${ }^{8}$

Dans l'étape de la «diplomatie des partis ", l'Europe a tissé cette trame de liens et de relations grâce à la diplomatie formelle, la solidarité politique et la coopération au développement, canalisant diverses initiatives et différents projets ponctuels, destinés essentiellement à la défense des droits de l'homme et au rétablissement des démocraties. Dans la nouvelle étape qui commence à se dessiner plus nettement pour les années 90, des instruments spécifiques de négociation, les manoeuvres électorales et les lobbies de pression qui permettent de structurer une politique économique, s'imposent sous le signe des « relations internationales de marché »".

Dans l'ensemble du sous-continent latino-américain, il est à souligner que, parmi les options actuelles de négociation ou de traité spécifique de la CEE pour la région, on peut prévoir sept théâtres possibles de négociation spécifique : les Caraïbes (les pays ACP de la convention de Lomé), l'Amérique centrale et Panama, le Groupe andin, le Mercosur, le Chili, le Mexique et Cuba. Tous ces espaces de négociation couvriraient une gamme variée d'intérêts et de motivations, allant d'un rapprochement étroit basé sur des motivations économiques, à des motivations politiques, et même, dans le cas extrême, à l'absence de négociations induisant des conditionnements politiques spécifiques, comme c'est le cas pour $\mathrm{Cuba}^{10}$.

Par ailleurs, si l'on tente d'élaborer un agenda prospectif et ponctuel de secteurs ou de domaines ayant les meilleures possibilités de négociation entre l'Amérique latine et l'Europe communautaire, on pourrait mentionner très brièvement: science et technologie, télécommunications, environnement, formation professionnelle, tourisme, culture, intégration dans son sens global - c'est-à-dire, dans ses diverses modalités et dimensions, telle qu'elle est en train de prendre forme en Amérique latine et dans les Caraïbes - commerce et financement du développement.

37 Tous les thèmes énoncés, et quelques autres comme la dette extérieure ou l'impact socioéconomique des politiques d'ajustement sur le développement de l'Amérique latine, doivent être traités de manière nouvelle, et non d'après les schémas traditionnels réservés à ces secteurs. Ils doivent, par conséquent, être adaptés aux mutations globales qu'a subies et subit encore le système actuel de relations internationales. Sans aucun doute, il faut chercher de nouveaux acteurs qui puissent assumer un nouveau contenu; trouver les créneaux les plus locaux et les plus ponctuels, ayant un impact concret et localisé, mais qui permettent l'interrelation des deux acteurs des sous-systèmes; autrement dit, viser à des liens sous étatiques qui présentent un dynamisme beaucoup plus large. En principe, l'éventail de choix et de possibilités n'est pas épuisé, bien que le chemin paraisse difficile à parcourir. Les deux parties ont peut-être adopté des points de vue plus traditionnels qui n'ont pas aidé au rapprochement des divers acteurs. 
38 L'Amérique latine ne peut oublier qu'il est indispensable pour elle de développer sa capacité de négociation. Et il faut bien se rendre compte qu'aucun pays latino-américain ne dispose, seul, de la capacité de négociation suffisante pour maximaliser les résultats des contacts avec les grands pôles de pouvoir. La réponse à l'Initiative pour les Amériques et à l'Europe d'après 92 est l'intégration latino-américaine qui développerait les possibilités et permettrait de passer des accords multilatéraux regroupant la région, afin d'atteindre une position de négociation plus avantageuse dans le nouvel ordre mondial.

\section{Réflexion à propos de Cuba et de l'intégration européenne}

Comme nous l'avons déjà signalé, Cuba est le seul pays latino-américain n'ayant pas d'accord-cadre qui réglemente et protège ses relations avec la CEE. Étant donné le dédale des relations entre Cuba et la CEE, la perspective de négociations plus fructueuses s'ouvrira lorsque la CEE aura éliminé les conditionnements politiques qui ont assombri le panorama des relations mutuelles. Le cas de Cuba doit être étudié par la CEE à la lumière d'une spécificité qui le différencie du reste des négociations établies, bien que, d'autre part, pour toute une série de thèmes, il coïncide avec les problèmes généraux susceptibles de se présenter dans les relations entre l'Europe communautaire et l'Amérique latine. Cuba constitue une synthèse d'éléments singuliers et communs au contexte et aux racines latino-américaines. Et ce n'est que dans cette perspective que les négociations pourront progresser de manière constructive. Par conséquent, un accord-cadre est indispensable en tant que base juridique pour entamer et élargir les relations fructueuses entre Cuba et les Communautés européennes.

Tout au long de l'histoire des contacts de l'Europe avec l'Amérique latine à ses différents niveaux d'exécution, Cuba a montré son intention de soutenir les initiatives qui aboutissent à un rapprochement de ces régions, avec un nombre incalculable d'éléments qui les rapprochent et les complètent. La position cubaine a toujours été favorable aux processus qui visent l'unité latino-américaine, car il n'est pas possible d'imaginer un processus différent de plates-formes et de réunions de contacts économiques, politiques et techniques qui, à notre avis, disperseraient les efforts, la superposition des forums de négociation et la présence de pays dans certains, mais pas dans d'autres. Cuba prône des actions conjointes pour que les Latino-Américains puissent se présenter avec des positions plus fortes et plus cohérentes. Si l'Amérique latine se divise, ses possibilités d'articulation aux défis des nouvelles conditions internationales seront également dispersées.

41 Dans cette logique, tout semble indiquer que le meilleur cadre pour maximaliser le dialogue le plus large avec les occidentaux, de manière efficace et réaliste, serait le système économique latino-américain, qui dispose d'une expérience et d'un appui technique suffisants pour essayer d'obtenir une concertation positive au bénéfice de tous.

Ces réflexions finales nous amènent à considérer l'avenir des relations entre Cuba et l'Europe communautaire comme étroitement lié aux possibilités réelles de modifier les tendances fondamentales qui influencent le développement de ce sous-système dans les années 90. L'actuelle conjoncture internationale n'est pas favorable à Cuba, et ce fait, s'ajoutant aux tensions qui découlent du différend entre Cuba et les États-Unis, offre un bilan peu encourageant, surtout si l'on considère le poids des pressions nord-américaines 
sur les agissements européens dans cette partie du monde ; la marge de manoeuvre de l'Europe en Amérique latine est bien définie afin de ne pas contrarier les desseins de la politique nord-américaine dans la région.

\section{NOTES}

1. Voir Fausto Buguèro Lomelé, «Nuevo orden : situacion y retos" in Problemas de Desarrollo, vol. XXII, México D.F., avril-juin 1991.

2. «Consideraciones sobre el estilo de las relaciones entre América Latina y el Caraibe con la comunidad Europea », Réunion de consultants, CE/SELA, Di. n 1, Caracas, 16-18 septembre 1991.

3. Des accords ont déjà été signés avec le Mexique, le Paraguay, l'Uruguay, l'Argentine et le Chili ; une négociation est en cours avec le Groupe andin.

4. Rapport de la CEPAL 1990.

5. Voir Alberto Van Klaveren, «Las relaciones europeo-latinoamericanas en el nuevo contexto internacional ", in Anuario de Politicas Exteriores Latinoamericanas 1990-1991, PROSPEL, Ed. Nueva Sociedad, Caracas, 1991.

6. Heine, J., « Relaciones europeo-latinoamericanas. Notas de Paz », Commission sud-américaine pour la paix, Santiago du Chili, avril 1992.

7. Walhelmy, M., «Las politicas latinoamericanas hacia Europa Occidental», Document de travail, $n^{\circ}$ 6, IRELA, 1987.

8. Dans cette partie du travail, nous suivons de près la position de Jorge Heine, "Cooperacion o divergencia ?Hacia una nueva agenda en las relaciones euro-latinoamericanas ", LC/R 929, CEPAL, Sem. 59/5, octobre 1990.

9. Ibidem

10. Cuba est actuellement le seul pays latino-américain à ne pas avoir d'accord; les actions ponctuelles de la Commission communautaire ont même été paralysées à deux reprises, essentiellement pour des raisons d'ordre politique.

\section{RÉSUMÉS}

The autor presents a study of the relations between Latin America and Europe in the ambit of a triangular relation that includes, besides these two countries, the United States that plays an essential role in these relations, on an economic and political level. The autor also insists on the paradox of relations between the two regions that consolidaded their political strenght, wereas they remain characterised by a reciprocal marginalisation if not a deterioration on aneconomic level. He puts forward the idea of a progressive replacement of a «diplomacy of parties » by « international market relations » as a guideline for european policy concerning Latin America. Furthermore, the reinforcement of the negociations on a regional or multilateral basis is considered as a fundamental means to reinforce the power of negociation of Latin American 
countries faced with a Europe in process of economic and political unification. In the last part, the autor briefly explains the specificity of relations between Cuba and the European $\mathrm{C}$ ommunity.

\section{AUTEUR}

VERONICA LOYNAZ

Centre d'études sur l'Amérique, LaHavane, Cuba 